\title{
On Complex Quadratic Fields with Class-Number Two
}

\author{
By H. M. Stark
}

To D. H. and Emma Lehmer

\begin{abstract}
Let $d<0$ be the discriminant of a complex quadratic field of class-number $h(d)$. In a previous paper the author has effectively shown how to find all $d$ with $h(d)=2$. In this paper, it is proved that, if $h(d)=2$, then $|d| \leqslant 427$.
\end{abstract}

1. Introduction. The work for this paper was completed in the summer of 1971 and reported on then in various places, including the informal conference at Asilomar organized by the Lehmers. It is particularly appropriate that it appear here since it is a direct generalization of my Ph.D. thesis, which was written under the supervision of D. H. Lehmer. Let $d<0$ be the discriminant of a complex quadratic field of classnumber $h(d)$. The aim of this paper is to prove

THEOREM 1. If $h(d)=2$, then $d$ is one of the eighteen numbers $-15,-20$, $-24,-35,-40,-51,-52,-88,-91,-115,-123,-148,-187,-232,-235,-267$, $-403,-427$.

Actually, we have proved in [3] that if $h(d)=2$, then $|d|<10^{1030}$. Thus it suffices to prove the following result:

THEOREM 2. If $h(d)=2$ and $|d|<10^{2000}$, then $d$ is one of the eighteen numbers listed in Theorem 1.

Outside of a result of Lehmer, Lehmer and Shanks quoted in Lemma 5, every number used in this paper was calculated in 1971 on a programable desk calculator. ${ }^{*}$ Indeed, if we restrict ourselves to the range $4 \cdot 10^{11}<|d|<10^{2000}$ (as Lemma 5 almost lets us do) and if we accept the numbers in Table 1 (certain constants involving the first 11 zeros of $\zeta(s)$ ), then we shall see that there is only one point that even needs a programable calculator (Lemma 11 below). All the other results regarding the range $4 \cdot 10^{11}<|d|<10^{2000}$ have been written so that they may be verified by hand. Since this work was first announced, Montgomery and Weinberger [2] have al-

Received August 1, 1974.

AMS (MOS) subject classifications (1970). Primary 10H05, 12A25, 12 A50; Secondary 10H10, $12 \mathrm{~A} 70$.

Key words and phrases. Class-number, quadratic field, binary quadratic forms, zeta functions.

*Incidentally, all of these numbers have been calculated twice on different machines with an interval of three years between calculations. Both sets of calculations are in complete agreement. 
so proved Theorem 1. Although similar in spirit, their method involves zeros of $L$ functions with large conductors. The numbers in their tables depend on an extended computer calculation.

\section{Theoretical Preliminaries. Let}

$$
Q(m, n)=a m^{2}+b m n+c n^{2}, \quad d=b^{2}-4 a c<0, \quad a>0,
$$

be a positive definite quadratic form and let

$$
\zeta(s, Q)=\frac{1}{2} \sum_{m, n \neq 0,0} Q(m, n)^{-s} .
$$

We let $B_{k}(x)$ be the $k$ th Bernoulli polynomial $\left(B_{0}(x)=1, B_{1}(x)=x-1 / 2, B_{2}(x)=\right.$ $\left.x^{2}-x+1 / 6, \cdots\right)$. We will have occasion to use $B_{k}(x-[x])$ where $[x]$ is the greatest integer function. The greatest integer notation occurs only with the Bernoulli polynomials. By the Euler-Maclaurin sum formula

$$
\begin{aligned}
& \zeta(s, Q)=\sum_{m=1}^{\infty}\left(a m^{2}\right)^{-s}+a^{-s} \sum_{n=1}^{\infty} \sum_{m=-\infty}^{\infty}\left[\left(m+\frac{b n}{2 a}\right)^{2}+\frac{|d|}{4 a^{2}} n^{2}\right]^{-s} \\
& =a^{-s} \zeta(2 s)+a^{-s} \sum_{n=1}^{\infty}\left\{\int_{-\infty}^{\infty}\left[\left(x+\frac{b n}{2 a}\right)^{2}+\frac{|d|}{4 a^{2}} n^{2}\right]^{-s} d x\right. \\
& \left.+\int_{-\infty}^{\infty} B_{1}(x-[x]) \frac{d}{d x}\left(\left[\left(x+\frac{b n}{2 a}\right)^{2}+\frac{|d|}{4 a^{2}} n^{2}\right]^{-s}\right) d x\right\} \\
& =a^{-s} \zeta(2 s)+2^{2 s-1} a^{s-1}|d|^{(1 / 2)-s} \zeta(2 s-1) \int_{-\infty}^{\infty}\left(u^{2}+1\right)^{-s} d u+h(s, Q)
\end{aligned}
$$

where

$$
h(s, Q)=a^{-s} \sum_{j=1}^{\infty} \int_{-\infty}^{\infty} B_{1}(x-[x]) \frac{d}{d x}\left\{\left[\left(x+\frac{b j}{2 a}\right)^{2}+\frac{|d|}{4 a^{2}} j^{2}\right]^{-s}\right\} d x .
$$

With $s=\sigma+i t$, we proved in [4] that for $\sigma \geqslant 1 / 2, k \geqslant 2, k$ even, $|d| / a^{2} \geqslant 4$,

$$
|h(s, Q)|<a^{-1 / 2} 2 \pi\left(\frac{k}{k-1}\right)^{2}\left(\frac{4|s|+2 k-2}{\pi\left(2|d| a^{-2}\right)^{1 / 2}}\right)^{k} \text {. }
$$

For our purposes here, we need to improve this estimate.

LEMma 1 . If $\sigma \geqslant 1 / 2, k \geqslant 2$, then

$$
|h(s, Q)|<2\left(\frac{4 a}{|d|}\right)^{\sigma-1 / 2}\left(\frac{2 \pi}{a k}\right)^{1 / 2}\left(\frac{k}{k-1}\right)^{2}\left[\frac{2|s|+(k-1) / 2}{\pi\left(|d| / a^{2}\right)^{1 / 2}}\right]^{k} \exp \left(\frac{1}{4 k}+\frac{1}{2 \pi^{3} k^{2}}\right)
$$

Proof. As in [4], we let $x+b j /(2 a)=u j|d|^{1 / 2} /(2 a)$ give a change of variable from $x$ to $u$ and then we integrate by parts $k-1$ times to get 
(2)

$$
\begin{aligned}
& \int_{-\infty}^{\infty} B_{1}(x-[x]) \frac{d}{d x}\left\{\left[\left(x+\frac{b j}{2 a}\right)^{2}+\frac{|d|}{4 a^{2}} j^{2}\right]^{-s}\right\} d x \\
& =(-1)^{k-1}\left(\frac{2 a}{j|d|^{1 / 2}}\right)^{k+2 s-1} \int_{-\infty}^{\infty} \frac{B_{k}(x-[x])}{k !} \cdot \frac{d^{k}}{d u^{k}}\left\{\left(u^{2}+1\right)^{-s}\right\} d u .
\end{aligned}
$$

As in [4], we use the estimate,

$$
\left|B_{k}(x) / k !\right| \leqslant 2 \zeta(k) /(2 \pi)^{k}
$$

but we will improve our estimate of $\left(d^{k} / d u^{k}\right)\left\{\left(u^{2}+1\right)^{-s}\right\}$. Let

$$
g_{k}(s, u)=(-1)^{k}\left(u^{2}+1\right)^{s+k / 2}\left(d^{k} / d u^{k}\right)\left\{\left(u^{2}+1\right)^{-s}\right\} .
$$

Thus $g_{k}(s, u)$ is a polynomial in $s$ and a rational function of $u$ and $\left(u^{2}+1\right)^{1 / 2}$. For small $y$ and $u$, we have

$$
\left(\frac{y^{2}+1}{u^{2}+1}\right)^{-s}=\sum_{k=0}^{\infty}(-1)^{k} g_{k}(s, u) \frac{(y-u)^{k}}{k !\left(u^{2}+1\right)^{k / 2}},
$$

the left-hand side being given by the principal value. If we let

$$
u=\operatorname{cotan} \theta, \quad 0<\theta<\pi,
$$

and

$$
y-u=-\left(u^{2}+1\right)^{1 / 2} z
$$

then the expansion (4) becomes

$$
\left[\left(1-e^{i \theta} z\right)\left(1-e^{-i \theta} z\right)\right]^{-s}=\sum_{k=0}^{\infty} g_{k}(s, u) \frac{z^{k}}{k !}
$$

which is valid for small $z$ and $\theta$ near $\pi / 2$. On the other hand, for small $z$, we may get this by multiplying the series for $\left(1-e^{i \theta} z\right)^{-s}$ and $\left(1-e^{-i \theta} z\right)^{-s}$; in this way we get

$$
g_{k}(s, u)=\sum_{j=0}^{k}\left(\begin{array}{l}
k \\
j
\end{array}\right)[s(s+1) \cdots(s+j-1)][s(s+1) \cdots(s+k-j-1)] e^{i(k-2 j) \theta} \text {. }
$$

This is now valid by analytic continuation for all $s$ and all real $u$ (i.e., all $\theta$ in the range $0<\theta<\pi$ ). By putting absolute value signs everywhere in (5), we see that $\left|g_{k}(s, u)\right|$ is less than or equal to the coefficient of $z^{k} / k$ ! in

$$
[(1-z)(1-z)]^{-|s|}=(1-z)^{-2|s|}
$$

and hence for all $s$ and all real $u$,

$$
\begin{aligned}
\left|g_{k}(s, u)\right| & \leqslant|2 s|(|2 s|+1) \cdots(|2 s|+k-1) \\
& <[|2 s|+(k-1) / 2]^{k} .
\end{aligned}
$$

Using this and (2), we find for $\sigma \geqslant 1 / 2$ that

$$
|h(s, Q)|<2 a^{-\sigma} \zeta(k)^{2}\left(\frac{2 a}{|d|^{1 / 2}}\right)^{2 \sigma-1}\left[\frac{2|s|+(k-1) / 2}{\pi\left(|d| / a^{2}\right)^{1 / 2}}\right]^{k} \int_{-\infty}^{\infty}\left(u^{2}+1\right)^{-(k+1) / 2} d u
$$

To simplify this, we note that 
Now Stirling's formula,

$$
\int_{-\infty}^{\infty}\left(u^{2}+1\right)^{-(k+1) / 2} d u=\frac{\pi^{1 / 2} \Gamma(k / 2)}{\Gamma((k+1) / 2)} .
$$

$$
\begin{aligned}
\log \Gamma(z)= & (z-1 / 2) \log z-z+1 / 2 \log (2 \pi) \\
& +\frac{B_{2}(0)}{2 z}-\frac{B_{3}(0)}{6 z^{2}}-\frac{1}{3} \int_{0}^{\infty} \frac{B_{3}(u-[u])}{(u+z)^{3}} d u
\end{aligned}
$$

and the same generalization used in [4],

$$
\begin{aligned}
\log \Gamma(z+1 / 2)= & z \log z-z+1 / 2 \log (2 \pi) \\
& +\frac{B_{2}(1 / 2)}{2 z}-\frac{B_{3}(1 / 2)}{6 z^{2}}-\frac{1}{3} \int_{0}^{\infty} \frac{B_{3}(u-1 / 2-[u-1 / 2])}{(u+z)^{3}} d u
\end{aligned}
$$

shows that for real $z>0$,

$$
\log \frac{\Gamma(z)}{\Gamma(z+1 / 2)}<-1 / 2 \log z+\frac{1}{8 z}+\frac{1}{3} \cdot 2 \cdot \frac{2 \zeta(3)}{(2 \pi)^{3}} \cdot \frac{1}{2 z^{2}} .
$$

We set $z=k / 2$ in this and then use the estimate $\zeta(m)<m /(m-1)$ for $m=3$ and $m=k$; the lemma follows.

Lemma 2. Suppose that $a \leqslant(|d| / 3)^{1 / 2}$ and that $\sigma=1 / 2$. For all integers $J \geqslant 1$, we have

$$
|h(s, Q)|<4|s|(3|d|)^{-1 / 4}\left[\sum_{j=1}^{J-1} j^{-1}+\frac{|2 s+1|}{3 \sqrt{3}} \sum_{j=J}^{\infty} j^{-2}\right] .
$$

Proof. For $\sigma=1 / 2$, we have

$$
\begin{aligned}
& \left|\int_{-\infty}^{\infty} B_{1}(x-[x]) \frac{d}{d x}\left\{\left[\left(x+\frac{b j}{2 a}\right)^{2}+\frac{|d|}{4 a^{2}} j^{2}\right]^{-s}\right\} d x\right| \\
& \quad \leqslant|s| \int_{-\infty}^{\infty} \frac{1}{2}\left(y^{2}+\frac{|d|}{4 a^{2}} j^{2}\right)^{-3 / 2} \cdot 2|y| d y \\
& \leqslant 2|s|\left(\frac{|d|}{4 a^{2}} j^{2}\right)^{-1 / 2}
\end{aligned}
$$

On the other hand, we may integrate by parts as in Lemma 1. For this purpose, we note that

$$
\left(d^{2} / d u^{2}\right)\left[\left(u^{2}+1\right)^{-s}\right]=2 s\left(u^{2}+1\right)^{-s-1}\left[(2 s+1)-\left(u^{2}+1\right)^{-1}(2 s+2)\right] .
$$

As $u$ runs from 0 to $\infty,(2 s+1)-\left(u^{2}+1\right)^{-1}(2 s+2)$ covers the straight line segment from -1 to $(2 s+1)$. Thus for $\sigma=1 / 2$,

$$
\left|\left(d^{2} / d u^{2}\right)\left[\left(u^{2}+1\right)^{-s}\right]\right|<|2 s(2 s+1)|\left(u^{2}+1\right)^{-3 / 2} .
$$

It follows from (2) with $k=2$ and $\sigma=1 / 2$ that 


$$
\begin{gathered}
\left|\int_{-\infty}^{\infty} B_{1}(x-[x]) \frac{d}{d x}\left\{\left[\left(x+\frac{b j}{2 a}\right)^{2}+\frac{|d|}{4 a^{2}} j^{2}\right]^{-s}\right\} d x\right| \\
\leqslant\left(\frac{2 a}{j|d|^{1 / 2}}\right)^{2} \int_{-\infty}^{\infty} \frac{1}{12}\left|\frac{d^{2}}{d u^{2}}\left[\left(u^{2}+1\right)^{-s}\right]\right| d u \\
\leqslant \frac{4 a^{2}}{|d| j^{2}} \cdot \frac{|2 s(2 s+1)|}{12} \int_{-\infty}^{\infty}\left(u^{2}+1\right)^{-3 / 2} d u \\
\leqslant 4 a^{2}|s(2 s+1)| /\left(3|d|^{2}\right) .
\end{gathered}
$$

We use the estimate (7) for $j \leqslant J-1$ and the estimate (8) for $j \geqslant J$ to get

$$
|h(s, Q)| \leqslant \frac{4 a^{1 / 2}|s|}{|d|^{1 / 2}} \sum_{j=1}^{J-1} j^{-1}+\frac{4 a^{3 / 2}|s(2 s+1)|}{3|d|} \sum_{j=J}^{\infty} j^{-s} .
$$

The right-hand side increases with $a$ and so we may replace $a$ by $(|d| / 3)^{1 / 2}$. This gives (6).

Lemma 3. Let $Q(x, y)$ be a reduced quadratic form. Then for integral $x$ and $y$ not both zero, $Q(x, y) \geqslant a$ and if $y \neq 0, Q(x, y) \geqslant c$.

Proof. We have

$$
4 a Q(x, y)=(2 a x+b y)^{2}+|d| y^{2}
$$

and since $Q(x, y)$ is reduced, $c \geqslant a \geqslant|b|$. Thus for $|y| \geqslant 2$,

while for $|y|=1$,

$$
Q(x, y) \geqslant|d| / a=4 c-b^{2} / a \geqslant 4 c-a>c,
$$

$$
Q(x, y) \geqslant\left(b^{2}+|d|\right) / 4 a=c
$$

and finally for $y=0, x \neq 0$,

$$
Q(x, y) \geqslant a x^{2} \geqslant a
$$

From this point on, $d<0$ is a discriminant of a quadratic field of class-number two. There are then two reduced quadratic forms of discriminant $d$; the principal form is

$$
Q_{1}(x, y)= \begin{cases}x^{2}+\frac{|d|}{4} y^{2} & \text { if } d \text { is even, } \\ x^{2}+x y+\frac{|d|+1}{4} y^{2} & \text { if } d \text { is odd }\end{cases}
$$

and the other reduced form will be denoted throughout by

$$
Q_{2}(x, y)=a x^{2}+b x y+c y^{2} .
$$

Thus $a \leqslant(|d| / 3)^{1 / 2}$ since $Q_{2}$ is reduced and $a$ is a prime number by Lemma 3 and the fact that some prime factor of $a$ must be represented by $Q_{2}$. If $c \neq a$ then either $b=0$ or $b=a$. In either event $a \mid d$ and $a \nmid c$, since then $d$ could not be a field discriminant. But some prime factor of $c$ must be represented by $Q_{2}$ and so $c$ is also a prime. 
Lemma 4. Let $p=p_{1}$ and $p=p_{2}\left(p_{1}<p_{2}\right)$ be the smallest primes such that $(d / p) \neq-1$. Then $p_{1}=a$ and if $c \neq a$ then $p_{2}=c$. If $p_{1}=a<(|d| / 4)^{1 / 2}$, then ald and $c>(|d| / 4)^{1 / 2}$. If $p$ is a prime and $(d / p)=1$, then $p>(|d| / 4)^{1 / 2}$.

Proof. By Lemma 3, any prime represented by $Q_{1}$ must be $\geqslant|d| / 4$. We know that $a$ and $c$ are both primes (sometimes equal). Further since $|d| \geqslant 15, a<$ $(|d| / 3)^{1 / 2}<|d| / 4$ and

$$
c=\frac{b^{2}+|d|}{4 a} \leqslant \frac{a}{4}+\frac{|d|}{4 a}<\frac{1}{4}\left(\frac{|d|}{3}\right)^{1 / 2}+\frac{|d|}{8}<\frac{|d|}{4}
$$

we see from Lemma 3 that $p_{1}=a$ and if $a \neq c$, then $p_{2}=c$. If $a<(|d| / 4)^{1 / 2}$, then $c>|d| /(4 a)>(|d| / 4)^{1 / 2}$. The rest of the lemma follows.

Lemma 4 allows us to very efficiently search through "small" values of $d$ for fields of class-number two. Fortunately a search for something similar is in the literature and can be adapted to our purposes.

Lemma 5. Let $M_{a}$ be the smallest positive integer such that $M_{a} \equiv 3(\bmod 8)$ and such that for all primes $p<a,\left(-M_{a} / p\right)=-1$. Then

$$
M_{53}=1333963, \quad M_{67}=20950603, \quad M_{149}=575528148427 \text {. }
$$

If $a_{0}=53,67$, or 149 and $a \geqslant a_{0}$ then $|d|>M_{a_{0}}$.

Proof. The values of $M_{a}$ are part of Table 3 of [1]. Note that if $p$ is the largest prime less than $a$, our $M_{a}$ is the $N_{p}$ of [1]. Dr. Shanks has kindly informed me that the value of $N_{149}$ (our $M_{151}$ ) is erroneous and that it should be increased to $N_{149}=N_{151}$. This does not affect the values that we use here. When $d$ is even, $a=2$. When $d$ is odd, $a=2$ only for $d=-15$. Otherwise for odd $d, a>2$ and this gives $(d / 2)=-1$ which implies $|d| \equiv 3(\bmod 8)$. Thus by Lemma 4 , if $a \geqslant a_{0}$ then $|d| \geqslant M_{a_{0}}$.

For $a \geqslant 149$, Lemma 5 gives us a convenient jumping off place. Unfortunately, for smaller $a$, the starting point is not so advanced. For $a<53$, [1] does us no good at all since $M_{47}=77683$, which is far too small to use in our present estimates (and $\left.M_{41}=163\right)$. In the sequel, what we will actually use is

$$
M_{149}>4 \cdot 10^{11}, \quad M_{67}>6 \cdot 10^{6}, \quad M_{53}>1.2 \cdot 10^{6} .
$$

3. Some Numerical Estimates. For convenience, we let $h_{j}(s)=h\left(s, Q_{j}\right), j=1,2$. Also we let

$$
L_{d}(s)=\sum_{n=1}^{\infty}\left(\frac{d}{n}\right) n^{-s}
$$

We then have,

$$
\begin{aligned}
\zeta(s) L_{d}(s)= & \zeta\left(s, Q_{1}\right)+\zeta\left(s, Q_{2}\right) \\
= & \left(1+a^{-s}\right) \zeta(2 s)+\left(1+a^{s-1}\right)\left(\frac{|d|}{4}\right)^{1 / 2-s} \cdot \frac{\pi^{1 / 2} \Gamma(s-1 / 2)}{\Gamma(s)} \zeta(2 s-1) \\
& +h_{1}(s)+h_{2}(s) .
\end{aligned}
$$

After an application of the functional equation of $\zeta(s)$, we get 


$$
\begin{aligned}
\zeta(s) L_{d}(s)-\left(1+a^{-s}\right) \zeta(2 s)= & \left(1+a^{s-1}\right) \zeta(2-2 s) \frac{\Gamma(1-s)}{\Gamma(s)}\left(\frac{|d|}{4 \pi^{2}}\right)^{1 / 2-s} \\
& +h_{1}(s)+h_{2}(s) .
\end{aligned}
$$

We apply this equation with $s=\rho_{m}=1 / 2+i \gamma_{m}$, a zero of $\zeta(s)$. The result is

$$
\left(\frac{|d|}{4 \pi^{2}}\right)^{i \gamma_{m}}=-\frac{\left(1+a^{-1 / 2+i \gamma_{m}}\right) \zeta\left(1-2 i \gamma_{m}\right) \Gamma\left(1 / 2-i \gamma_{m}\right)}{\left(1+a^{-1 / 2-i \gamma_{m}}\right) \zeta\left(1+2 i \gamma_{m}\right) \Gamma\left(1 / 2+i \gamma_{m}\right)}
$$

$$
-\left(\frac{|d|}{4 \pi^{2}}\right)^{i \gamma_{m}} \cdot \frac{h_{1}\left(\rho_{m}\right)+h_{2}\left(\rho_{m}\right)}{\zeta\left(2 \rho_{m}\right)\left(1+a^{-\rho_{m}}\right)}
$$

Let $\alpha_{m}, 0 \leqslant \alpha_{m}<2 \pi$ be defined by

$$
\alpha_{m} \equiv \pi-2 \arg \zeta\left(2 \rho_{m}\right)-2 \arg \Gamma\left(\rho_{m}\right) \quad(\bmod 2 \pi) .
$$

For convenience we let

$$
\delta_{m}(a)=\frac{\left|h_{1}\left(\rho_{m}\right)\right|+\left|h_{2}\left(\rho_{m}\right)\right|}{\left(1-a^{-1 / 2}\right)\left|\zeta\left(2 \rho_{m}\right)\right|}
$$

It will also be useful to let $\theta$ denote a number, not necessarily the same each time it occurs, such that $|\theta| \leqslant 1$.

LEMмA 6. If $\delta_{m}(a)<1 / 2$ then there is an integer $x_{m}$ such that

$$
\gamma_{m} \log \left(\frac{|d|}{4 \pi^{2}}\right)=\alpha_{m}+2 \pi x_{m}+2 \arg \left(1+a^{-1 / 2+i \gamma_{m}}\right)+\frac{\pi}{3} \delta_{m}(a) \theta .
$$

Proof. The lemma follows from (9) thanks to the elementary inequality,

which is valid for $|z| \leqslant 1 / 2$.

$$
|\arg (1+z)| \leqslant \pi|z| / 3
$$

Now we define

and

$$
A_{n}=\frac{1}{2 \pi}\left(\frac{\gamma_{n}}{\gamma_{1}} \alpha_{1}-\alpha_{n}\right)
$$

$$
B_{n}(a)=\frac{1}{\pi}\left[\frac{\gamma_{n}}{\gamma_{1}} \arg \left(1+a^{-1 / 2+i \gamma_{1}}\right)-\arg \left(1+a^{-1 / 2+i \gamma_{n}}\right)\right]
$$

(the Bernoulli polynomials will not occur again).

LEMMA 7. If $\delta_{m}(a)<1 / 2$ for $m=1$ and $m=n$, then

$$
x_{n}=\frac{\gamma_{n}}{\gamma_{1}} x_{1}+A_{n}+B_{n}(a)+\frac{\theta}{6}\left[\frac{\gamma_{n}}{\gamma_{1}} \delta_{1}(a)+\delta_{n}(a)\right] \text {. }
$$

If further $a \geqslant 4$, then

$$
x_{n}=\frac{\gamma_{n}}{\gamma_{1}} x_{1}+A_{n}+\frac{\theta}{6}\left[2 a^{-1 / 2}\left(\frac{\gamma_{n}}{\gamma_{1}}+1\right)+\frac{\gamma_{n}}{\gamma_{1}} \delta_{1}(a)+\delta_{n}(a)\right] \text {. }
$$

Proof. Equation (12) follows from Lemma 6 and (13) follows from (12) and (11). 
Now we need some explicit numerical estimates for $\left|h_{j}\left(\rho_{m}\right)\right|$ for various $m$. The nature of Lemmas 1 and 2 is such that any estimate derived for $h_{2}$ will automatically be valid for $h_{1}$ when the $a^{-1 / 2}$ term is deleted. We have,

$$
\left|h_{2}\left(\rho_{m}\right)\right|< \begin{cases}7.2 \cdot 10^{-11} a^{-1 / 2} & \text { for } m \leqslant 11 \text { and }|d| / a^{2} \geqslant 4000 \\ 3.3 \cdot 10^{-11} a^{-1 / 2} & \text { for } m \leqslant 2 \text { and }|d| / a^{2} \geqslant 800 \\ 1.043 a^{-1 / 2} & \text { for } m \leqslant 2 \text { and }|d| / a^{2} \geqslant 250\end{cases}
$$

These estimates follow from Lemma 1 with $|s|=55, k=84$ in the first, $|s|=21.05$, $k=44$ in the second and $|s|=21.05, k=11$ in the third. Corresponding to these are the estimates

$$
\left|h_{1}\left(\rho_{m}\right)\right|< \begin{cases}7.2 \cdot 10^{-11} & \text { for } m \leqslant 11 \text { and }|d| \geqslant 4000 \\ 3.3 \cdot 10^{-11} & \text { for } m \leqslant 2 \text { and }|d| \geqslant 800\end{cases}
$$

We also need some estimates from Lemma 2. With $s=\rho_{1}$ and $J=6$ in Lemma 2, we get

$$
\left|h_{2}\left(\rho_{1}\right)\right|<141|d|^{-1 / 4}
$$

and with $s=\rho_{2}, J=9$ in Lemma 2 we get

$$
\left|h_{2}\left(\rho_{2}\right)\right|<235|d|^{-1 / 4} \text {. }
$$

From these and the values of $\left|\zeta\left(2 \rho_{n}\right)\right|$ in Table 1 , we get without any difficulty,

$$
\left|\delta_{m}(a)\right|< \begin{cases}10^{-9} & \text { for } m \leqslant 11 \text { and }|d| / a^{2} \geqslant 4000, \\ 2.4 \cdot 10^{-10} & \text { for } m \leqslant 2,|d| / a^{2} \geqslant 800, \\ 2 \cdot 10^{-10}+1.26\left(a^{1 / 2}-1\right)^{-1} & \text { for } m=2,|d| / a^{2} \geqslant 250, \\ 10^{-10}+.537\left(a^{1 / 2}-1\right)^{-1} & \text { for } m=1,|d| / a^{2} \geqslant 250, \\ 2 \cdot 10^{-10}+283|d|^{-1 / 4}\left(1-a^{-1 / 2}\right)^{-1} & \text { for } m=2,|d| \geqslant 800, \\ 10^{-10}+72.5|d|^{-1 / 4}\left(1-a^{-1 / 2}\right)^{-1} & \text { for } m=1,|d| \geqslant 800 .\end{cases}
$$

LEMmA 8. If either $|d| / a^{2} \geqslant 800$, or $|d| / a^{2} \geqslant 250, a \geqslant 5$ or $|d|>10^{9}$, then

and

$$
x_{1}>2.249 \log |d|-8.543-\frac{1}{3} a^{-1 / 2} \text {, }
$$

$$
|d|>41.4 \exp \left(.444 x_{1}-.149 a^{-1 / 2}\right) \text {. }
$$

In particular, $x_{1}>3$ in all cases and if $x_{1}>10400,|d|>10^{2000}$.

Proof. We see from (18) that $\left|\delta_{1}(a)\right|<1 / 2$ under the hypotheses of the lemma (we only need to make this estimate in the third case for $a>100$ as otherwise the first case applies). We note that (11) holds for $z=a^{-1 / 2+i \gamma_{1}}$ (for $a=2$ and 3 by direct calculation) and thus Lemma 6 implies

$$
x_{1}>\frac{\gamma_{1}}{2 \pi} \log \left(\frac{|d|}{4 \pi^{2}}\right)-\frac{\alpha_{1}}{2 \pi}-\frac{1}{12}-\frac{1}{3} a^{-1 / 2}
$$


and

$$
|d|>4 \pi^{2} \exp \left[\frac{2 \pi x_{1}+\alpha_{1}-(\pi / 6)-(2 \pi / 3) a^{-1 / 2}}{\gamma_{1}}\right] .
$$

The lemma follows from the numerical values of $\gamma_{1}$ and $\alpha_{1}$ in Table 1 .

TABLE 1

\begin{tabular}{cccccc}
\hline$n$ & $\gamma_{n}+5 \cdot 10^{-10} \theta$ & $\alpha_{n} /(2 \pi)+10^{-7} \theta$ & $\zeta\left(2 \rho_{n}\right) \mid+10^{-4} \theta$ & $\gamma_{n} \gamma_{1}+10^{-7} \theta$ & $A_{n}+10^{-7} \theta$ \\
1 & 14.134725142 & .189940085 & 1.9488 & & \\
2 & 21.022039639 & .744277023 & .8310 & 1.487262004 & -.461786352 \\
3 & 25.010857580 & .644452141 & .5342 & 1.769461898 & -.308360397 \\
4 & 30.424876126 & .868568588 & .5148 & 2.152491528 & -.459724164 \\
5 & 32.935061588 & .424902705 & .8130 & 2.330081501 & .017673174 \\
6 & 37.586178159 & .399505477 & .9383 & 2.659137534 & .105571332 \\
7 & 40.918719012 & .353564641 & 1.9220 & 2.894907301 & .196294299 \\
8 & 43.327073281 & .439618184 & .9778 & 3.065292946 & .142603819 \\
9 & 48.005150881 & .380301171 & .5426 & 3.396256411 & .264784061 \\
10 & 49.773832478 & .365820574 & 1.4281 & 3.521386654 & .303031906 \\
11 & 52.970321478 & .266601822 & .6885 & 3.747531058 & .445204546 \\
\hline & & & & &
\end{tabular}

The values of $\gamma_{2} / \gamma_{1}$ and $A_{2}$ are correct to within $5 \cdot 10^{-10} \theta$.

LEMMA 9. If $|d| / a^{2} \geqslant 4000$, then for $2 \leqslant n \leqslant 11$,

$$
\left|x_{n}-\frac{\gamma_{n}}{\gamma_{1}} x_{1}-A_{n}\right|<10^{-9}+\frac{1}{3} a^{-1 / 2}\left(\frac{\gamma_{n}}{\gamma_{1}}+1\right) \text {. }
$$

If $|d| / a^{2} \geqslant 800$, then

and if also $a>4$, then

$$
\left|x_{2}-\frac{\gamma_{2}}{\gamma_{1}} x_{1}-A_{2}-B_{2}(a)\right|<10^{-10}
$$

$$
\left|x_{2}-\frac{\gamma_{2}}{\gamma_{1}} x_{1}-A_{2}\right|<\cdot 10^{-10}+.83 a^{-1 / 2} \text {. }
$$

If $|d| / a^{2} \geqslant 250$ and $a \geqslant 13$, then

$$
\left|x_{2}-\frac{\gamma_{2}}{\gamma_{1}} x_{1}-A_{2}-B_{2}(a)\right|<10^{-10}+.344\left(a^{1 / 2}-1\right)^{-1},
$$

$$
\left|x_{2}-\frac{\gamma_{2}}{\gamma_{1}} x_{1}-A_{2}\right|<10^{-10}+.344\left(a^{1 / 2}-1\right)^{-1}+.83 a^{-1 / 2} .
$$

If $|d|>4 \cdot 10^{11}$ and $a \geqslant 10^{4}$, then

$$
\left|x_{2}-\frac{\gamma_{2}}{\gamma_{1}} x_{1},-A_{2}\right|<10^{-10}+66|d|^{-1 / 4}+.83 a^{-1 / 2} \text {. }
$$


Proof. We see from (18) that $\delta_{m}(a)<1 / 2$ for $m=1$ and $m=n$ in every case. The lemma is now a direct consequence of (12) and (13) of Lemma 7 together with the estimates in (18).

4. Proof of Theorem 2. We now wish to systematically examine the inequalities of Lemma 9 and show that $x_{1}>10400$ so that by Lemma $8,|d|>10^{2000}$.

Lemma 10. If $a \geqslant 10^{14}$, then $|d|>10^{2000}$.

Proof. For $a \geqslant 10^{14}$, we have $|d|>3 \cdot 10^{28}$ (and by Lemma $8, x_{1}>3$ ). It follows from (24) that

$$
\left|x_{2}-\left(\gamma_{2} / \gamma_{1}\right) x_{1}-A_{2}\right|<5.15 \cdot 10^{-6}
$$

which we will write as

$$
\begin{gathered}
\left|\left(x_{2}-4\right)-\frac{5546}{3729}\left(x_{1}-3\right)-\left(\frac{\gamma_{2}}{\gamma_{1}}-\frac{5546}{3729}\right)\left(x_{1}-3\right)+.340 \cdot 10^{-6}\right| \\
<5.16 \cdot 10^{-6}
\end{gathered}
$$

since $4-\left(3 \gamma_{2} / \gamma_{1}+A_{2}\right)=.340 \cdot 10^{-6}+2 \cdot 10^{-9} \theta$ and

$$
\frac{\gamma_{2}}{\gamma_{1}}-\frac{5546}{3729}=(3+\theta) 10^{-9} \text {. }
$$

Therefore

$$
\left|\left(x_{2}-4\right)+\frac{5546}{3729}\left(x_{1}-3\right)\right|<6 \cdot 10^{-6}+4 \cdot 10^{-9} x_{1} .
$$

But for $x_{1} \leqslant 10400$, the right-hand side of (27) is less than $1 / 3729$ and so, since $(3729,5546)=1,3729 \mid\left(x_{1}-3\right)$. Thus if $x_{1}<10400$, then $x_{1}=3732$ or $x_{1}=$ 7461. But we see from (26) that $x_{1}=3732$ and $x_{1}=7461$ do not satisfy (25) either. Hence $x_{1}>10400$ and $|d|>10^{2000}$.

LEMmA 11. If $|d| / a^{2} \geqslant 4000$ and $a \geqslant 53$, then $|d|>10^{2000}$.

Proof. By Lemma $8, x_{1}>0$ and by (19) of Lemma 9,

$$
\left|x_{n}-\frac{\gamma_{n}}{\gamma_{1}} x_{1}-A_{n}\right|<\frac{1}{21}\left(\frac{\gamma_{n}}{\gamma_{1}}+1\right), \quad 2 \leqslant n \leqslant 11 .
$$

A check on a programable desk calculator shows this can not happen for $0<x_{1}<$ 10400 and this proves the lemma.

Incidentally $x_{1}=2324$ and $x_{1}=7898$ satisfy (28) for $2 \leqslant n \leqslant 10$. Perhaps the most interesting number though, is $x_{1}=42$ which satisfies eight of the ten inequalities in (28) and comes close on the other two. It would be interesting to know if somewhere around $-5 \cdot 10^{9}$ there is a discriminant with small class-number that accounts for this.

LEMma 12. If $53 \leqslant a<10^{14}$, then $|d|>10^{2000}$.

Proof. We break the interval on $a$ up into several pieces. We begin with $10^{4} \leqslant$ $a<10^{14}$. By Lemmas 5 and $8,|d|>4 \cdot 10^{11}$ and $x_{1}>51$. It follows from (24) that $x_{1} \geqslant 75$ (see Table 2) and therefore by Lemma $8,|d|>10^{16}$. For $10^{4} \leqslant a<$ 
TABLE 2. $\left|x_{2}-\left(\gamma_{2} / \gamma_{1}\right) x_{1}-A_{2}\right|+10^{-4} \theta$, where $x_{2}$ is the nearest integer to $\left(\gamma_{2} / \gamma_{1}\right) x_{1}+A_{2}$ for $0 \leqslant x_{1} \leqslant 85$

\begin{tabular}{c|cccccccccc}
\multicolumn{1}{c}{$x_{1}$} & 0 & 1 & 2 & 3 & 4 & 5 & 6 & 7 & 8 & 9 \\
\hline 0 & .4618 & .0255 & .4873 & .0000 & .4873 & .0255 & .4618 & .0510 & .4363 & .0764 \\
10 & .4108 & .1019 & .3854 & .1274 & .3599 & .1529 & .3344 & .1783 & .3089 & .2038 \\
20 & .2835 & .2293 & .2580 & .2548 & .2325 & .2802 & .2070 & .3057 & .1816 & .3312 \\
30 & .1561 & .3567 & .1306 & .3821 & .1051 & .4076 & .0796 & .4331 & .0542 & .4586 \\
40 & .0287 & .4840 & .0032 & .4905 & .0223 & .4650 & .0477 & .4395 & .0732 & .4141 \\
50 & .0987 & .3886 & .1242 & .3631 & .1496 & .3376 & .1751 & .3121 & .2006 & .2867 \\
60 & .2261 & .2612 & .2515 & .2357 & .2770 & .2102 & .3025 & .1848 & .3280 & .1593 \\
70 & .3534 & .1338 & .3789 & .1083 & .4044 & .0829 & .4299 & .0574 & .4554 & .0319 \\
80 & .4808 & .0064 & .4937 & .0190 & .4682 & .0445 & & & & \\
\hline
\end{tabular}

With an error of .0032 , the table is periodic in $x_{1}$ with period 39 . This is because $58 / 39$ is an excellent approximation to $\gamma_{2} / \gamma_{1}$. The small change from $x_{1}$ to $x_{1}+2$ is because $3-$ $2(58 / 39)=1 / 39$. These properties show that $\left|x_{2}-\left(\gamma_{2} / \gamma_{1}\right) x_{1}-A_{2}\right|$ will be small only at $x_{1}=3$, $42,81,120,159, \cdots$ and values removed from these by small even numbers.

$10^{6}$ we now have $|d| / a^{2}>4000$ and Lemma 11 applies. So we may restrict our attention to $10^{6} \leqslant a<10^{14}$ and $|d|>10^{16}$. But now by (24) again, $x_{1} \geqslant 81$ and by Lemma $8,|d|>16 \cdot 10^{16}$. Once more we apply (24), this time $x_{1}>160$ and so $|d|>10^{32}$ and $|d| / a^{2}>4000$. Therefore by Lemma $11,|d|>10^{2000}$.

Next, we take the range $149 \leqslant a<10^{4}$. By Lemma $5,|d|>4 \cdot 10^{11}$ and so by Lemma $11,|d|>10^{2000}$. Lastly, we take the range $53 \leqslant a<149$. By Lemma 5 , $|d|>1.2 \cdot 10^{6}$ and so by Lemma $8, x_{1}>22$. For $53 \leqslant a<67$ we have $|d| / a^{2}>$ 250 ; by Lemma 5 , for $67 \leqslant a<149,|d|>6 \cdot 10^{6}$ and again $|d| / a^{2}>250$. It follows from (23) that $x_{1} \geqslant 30$. By Lemma 8 , we now have $|d|>2.5 \cdot 10^{7}$. Hence $|d| / a^{2}>800$ and so by (21), $x_{1} \geqslant 34$. Therefore $|d|>10^{8}$ and so $|d| / a^{2}>4000$. Thus by Lemma $11,|d|>10^{2000}$ and this completes the proof.

We have now come to the point that we must make use of the numerical values of $B_{2}(a)$ with prime $a, 2 \leqslant a \leqslant 47$. $10^{2000}$

LEMmA 13. If $a<53$ and either $|d|>600000$ or $|d| / a^{2} \geqslant 800$, then $|d|>$

Proof. We recall that $a$ is a prime. For $|d|>600000$ and $a \leqslant 23$, we have $|d| / a^{2}>800$ already. For $|d|>600000$ and $29 \leqslant a \leqslant 47$, we have $x_{1}>21$ by Lemma 8 and $|d| / a^{2}>250$. By (22), together with the fact that $\left|B_{2}(a)\right|<.107$ for $29 \leqslant a \leqslant 47$ (see Table 3 ), we get $x_{1} \geqslant 28$ in this case which by Lemma 8 leads to $|d|>800 \cdot 47^{2}$ and so we have $|d| / a^{2}>800$ in all cases. Now by (20), 
TABLE 3

\begin{tabular}{ccc}
\hline$a$ & $B_{2}(a)+10^{-7} \theta$ & $3729 B_{2}(a)+10^{-3} \theta$ \\
\hline 2 & -.541421469 & -2018.961 \\
3 & .304504877 & 1135.499 \\
5 & -.355642421 & -1251.611 \\
7 & .176779814 & 659.212 \\
11 & .102601911 & 382.603 \\
13 & -.066432969 & -247.729 \\
17 & .084063829 & 313.474 \\
19 & -.038819482 & -144.758 \\
23 & .022308245 & 83.187 \\
29 & -.106959524 & -398.852 \\
31 & -.090143184 & -336.144 \\
37 & .026169905 & 97.588 \\
41 & .038194456 & 142.427 \\
43 & .048526374 & 180.955 \\
47 & -.034830231 & -129.882
\end{tabular}

or,

(29)

$$
\begin{aligned}
\mid 3729\left(x_{2}-4\right) & -5546\left(x_{1}-3\right)-3729 B_{2}(a) \\
& -3729\left(\frac{\gamma_{2}}{\gamma_{1}}-\frac{5546}{3729}\right)\left(x_{1}-3\right)-.001268 \mid<10^{-5} .
\end{aligned}
$$

If $x_{1}<10400$, then we see from (29) and (26) that

$$
\left|3729\left(x_{2}-4\right)-5546\left(x_{1}-3\right)-3729 B_{2}(a)\right|<.16 .
$$

From Table 3, we see that this is possible only for $a=2,29,31,43$ and 47. Further (29) and (26) eliminate $a=2,29$ and 47 (basically because the right-hand side of (26) is positive). This leaves $a=31$ and 43; in these cases (30) shows that we must have

This gives

$$
-5546\left(x_{1}-3\right) \equiv\left\{\begin{aligned}
-336(\bmod 3729) & \text { if } a=31 \\
181(\bmod 3729) & \text { if } a=43
\end{aligned}\right.
$$




$$
\begin{aligned}
& x_{1}=1095,4628,8553 \quad \text { if } a=31, \\
& x_{1}=2833,6562,10291 \quad \text { if } a=43 .
\end{aligned}
$$

When we put these values of $x_{1}$ in (29) and use (26), we see that (29) is not satisfied and so $x_{1}>10400$. Therefore $|d|>10^{2000}$.

In the case of $a=43$ and $x_{1}=2833$, we have

$$
\begin{aligned}
3729\left(4 \cdot 10^{-9}\right)\left(x_{1}-3\right) & =.0422+10^{-4} \theta, \\
3729 B_{2}(a) & =180.955+.001 \theta,
\end{aligned}
$$

and so the error term in (26) is essentially the maximum permissible. As a check, we could also try the above six values of $x_{1}$ in (12) of Lemma 7 with $n=3$; calculation reveals that the right-hand side of (12) is nowhere near an integer.

LEMMA 14. If $a \geqslant 13$, then $|d|>600000$.

Proof. By Lemma 4,

$$
(d / p)=-1 \quad \text { for } p=2,3,5,7,11 .
$$

There are precisely 30 possible values of $d(\bmod 9240)$ satisfying these conditions. For each of these thirty values, we then check the 64 or 65 values of $d$ between 0 and -600000 that are congruent to it $(\bmod 9240)$ and find that, besides $d=-67$, $d=-163$ (both class-number one) that every such $d$ is either divisible by two primes less than $(|d| / 4)^{1 / 2}$ or there is a prime $p<(|d| / 4)^{1 / 2}$ with $(d / p)=1$. Thus by Lemma 4 , if $a \geqslant 13$ then $|d|>600000$.

The search just described was easy on the desk calculator although it would have been a chore by hand. The search revealed that there are exactly two discriminants $d$ between 0 and -600000 satisfying (31) such that $d$ is divisible by at most one prime $\leqslant 47$ and is a nonresidue of all other primes $\leqslant 47$. These numbers are -85507 and -207883 . They do not contradict the value of $M_{53}$ in Lemma 5 since 37 divides the former and 13 divides the latter. Although their class-numbers are undoubtedly small for their size, they do not have class-number 2 since

$$
\left(\frac{-85507}{61}\right)=\left(\frac{-207883}{53}\right)=1 \text {. }
$$

Lemma 15. If $a=2,3,5,7,11$ then either $d$ is one of the eighteen discriminants in Theorem 1 or $|d|>800 a^{2}$.

Proof. It is easily checked that if $|d| \leqslant 484$, then $d$ is one of the eighteen numbers listed in Theorem 1 and so we assume that $|d|>484$ and so $(|d| / 4)^{1 / 2}>11$. Therefore by Lemma $4, a \mid d$ and $d$ is a nonresidue of the other four primes $\leqslant 11$. This gives us 30 possible values of $d(\bmod 4620)$ when $a=2$ and $30,15,10,6$ possible values of $d(\bmod 9240)$ when $a=3,5,7,11$ respectively. We now tabulate every value of $d$ between -484 and $-800 a^{2}$ satisfying these congruence conditions and such that if $p$ is a prime, $p \neq a, p<(|d| / 4)^{1 / 2}$ then $(d / p)=-1$. The list 
contains only nonfundamental discriminants (for example $-163 a^{2}$ for $a=2,3,5$ ). The lemma follows from Lemma 4.

Theorem 2 follows from Lemmas 10, 12, 13, 14 and 15 .

Mathematics Department

Massachusetts Institute of Technology

Cambridge, Massachusetts 02139

1. D. H. LEHMER, EMMA LEHMER \& DANIEL SHANKS, "Integer sequences having prescribed quadratic character," Math. Comp., v. 24, 1970, pp. 433-451. MR 42 \#5889.

2. H. L. MONTGOMERY \& P. J. WEINBERGER, "Notes on small class numbers," Acta Arith., v. 24, 1974, pp. 529-542.

3. H. M. STARK, "A transcendence theorem for class-number problems. II," Ann. of Math. (2), v. 96, 1972, pp. 174-209. MR $46 \# 8983$.

4. H. M. STARK, "On complex quadratic fields with class number equal to one," Trans. Amer. Math. Soc., v. 122, 1966, pp. 112-119. MR 33 \#4043. 